\title{
Behavioral Symptoms in Premanifest Huntington Disease Correlate with Reduced Frontal $\mathrm{CB}_{1} \mathrm{R}$ Levels
}

\author{
Jenny Ceccarini ${ }^{1}$, Rawaha Ahmad ${ }^{1}$, Laura Van de Vliet ${ }^{2,3}$, Cindy Casteels ${ }^{1}$, Mathieu Vandenbulcke ${ }^{2,3}$, \\ Wim Vandenberghe ${ }^{3,4}$, and Koen Van Laere ${ }^{1}$ \\ ${ }^{I}$ Nuclear Medicine and Molecular Imaging, University Hospitals Leuven, and Department of Imaging and Pathology, KU Leuven, \\ Leuven, Belgium; ${ }^{2}$ Department of Old Age Psychiatry, University Psychiatric Centre, KU Leuven, Leuven, Belgium; ${ }^{3}$ Department of \\ Neurosciences, KU Leuven, Leuven, Belgium; and ${ }^{4}$ Department of Neurology, University Hospitals Leuven, Leuven, Belgium
}

\begin{abstract}
Many Huntington disease (HD) mutation carriers already have cognitive and psychiatric symptoms in the premanifest (premotor) phase of the disease (pre-HD), but the molecular underpinnings of these symptoms are not well understood. Previous work has shown reduced availability of the cerebral type 1 cannabinoid receptor $\left(C_{1} R\right)$ in manifest $H D$. Here, we investigated whether $C_{1} R$ binding is related to cognitive and psychiatric symptoms in pre-HD mutation carriers. Methods: $\mathrm{CB}_{1} \mathrm{R}$ binding was measured with ${ }^{18} \mathrm{~F}-\mathrm{MK}-9470$ (N-[(2S,3S)-3-(3-cyanophenyl)-4-(4-ethoxyphenyl)butan-2-yl]-2methyl-2-(5-methylpyridin-2-yl)oxypropanamide) PET in 15 pre-HD subjects ( 8 men, 7 women; age, $39.3 \pm 9.9$ y), 15 gene-negative controls from HD families (9 men, 6 women; age, $37.0 \pm 10.6 \mathrm{y}$ ), and 12 community controls ( 6 men and 6 women; age, $39.9 \pm 15.1 \mathrm{y}$ ). All subjects also underwent extensive assessment of motor and cognitive function, as well as a behavioral test battery including the Problem Behavior Assessment for HD (PBA-HD), and MRI. Parametric binding images of ${ }^{18} \mathrm{~F}-\mathrm{MK}-9470$ were corrected for partial-volume effect. Results: There was no difference in $\mathrm{CB}_{1} \mathrm{R}$ binding, gray matter volume, cognitive function, or psychiatric scores between gene-negative controls from HD families and community controls, which were therefore pooled to one control group. Compared with controls, pre-HD subjects showed striatal atrophy, a decrease in $\mathrm{CB}_{1} \mathrm{R}$ binding in the prefrontal cortex, and higher PBA-HD scores on depression, apathy, and irritability (range, $P=$ $0.01-0.005)$. The PBA-HD scores inversely correlated with $C_{B} R$ binding in prefrontal regions and cingulate cortex in pre-HD (range: $r=-0.64$ to $-0.72 ; P=0.01-0.008$ ). Conclusion: The association between behavioral symptoms and reduced prefrontal $C_{1} R$ levels may provide new insight into the molecular basis of neuropsychiatric symptoms in pre-HD and suggest new therapeutic avenues.
\end{abstract}

Key Words: premanifest HD (pre-HD); cannabinoid type 1 receptor $\left(\mathrm{CB}_{1} \mathrm{R}\right)$; behavioral symptoms; PET

J Nucl Med 2019; 60:115-121

DOI: 10.2967/jnumed.118.210393

\section{$\mathbf{H}$} degenerative disorder caused by a CAG repeat expansion in the huntingtin $(H T T)$ gene. The toxic effects of mutant huntingtin

Received Feb. 23, 2018; revision accepted May 24, 2018.

For correspondence or reprints contact: Jenny Ceccarini, University Hospital Leuven, Herestraat 49, 3000 Leuven, Belgium.

E-mail: jenny.ceccarini@uzleuven.be

Published online Jun. 22, 2018.

COPYRIGHT (C 2019 by the Society of Nuclear Medicine and Molecular Imaging. protein induce neuronal cell loss, most prominently in the caudate nucleus and putamen but also in cortical regions. Clinically, HD patients develop motor dysfunction, cognitive decline, and psychiatric disturbances. Traditionally, the clinical diagnosis of HD is made when a carrier of the HD mutation develops motor signs that are typical of HD. However, many HD mutation carriers already have cognitive changes and psychiatric symptoms, such as depression, apathy, and irritability, in the premotor (premanifest) stage (pre-HD) (1-6). The molecular and anatomic origin of these preHD cognitive and psychiatric changes is not well understood.

The type 1 cannabinoid receptor $\left(\mathrm{CB}_{1} \mathrm{R}\right)$ is a crucial modulator of synaptic transmission in the brain and is involved in the regulation of motor behavior, cognition, and emotion (7). Several lines of evidence suggest an important role for $\mathrm{CB}_{1} \mathrm{R}$ in HD. In vivo PET imaging with ${ }^{18} \mathrm{~F}-\mathrm{MK}-9470(N-[(2 S, 3 S)-3-(3-c y a n o p h e n y l)-$ 4-(4-ethoxyphenyl)butan-2-yl]-2-methyl-2-(5-methylpyridin-2-yl) oxypropanamide) has revealed a substantial reduction of $\mathrm{CB}_{1} \mathrm{R}$ binding in patients with manifest $\mathrm{HD}$, even in early manifest stages (8), suggesting that the decline in $\mathrm{CB}_{1} \mathrm{R}$ availability may potentially already begin in the pre-HD phase. This hypothesis is supported by the observation that $\mathrm{CB}_{1} \mathrm{R}$ protein and messenger RNA levels are decreased in the pre-HD phase in transgenic mouse models of $\mathrm{HD}$, with a further decline during the manifest course of the disease (9). Also, genetic deletion of $\mathrm{CB}_{1} \mathrm{R}$ in transgenic HD mouse models aggravates symptomatology, suggesting that the decline of $\mathrm{CB}_{1} \mathrm{R}$ in human $\mathrm{HD}$ may actually underlie part of the clinical phenomenology.

So far, no human $\mathrm{CB}_{1} \mathrm{R}$ PET studies in pre-HD mutation carriers have been reported. In this study, we assessed in vivo $C_{1} R$ availability using ${ }^{18} \mathrm{~F}-\mathrm{MK}-9470 \mathrm{PET}$ in pre-HD mutation carriers in comparison to healthy, age-matched controls. To control for potential effects of the distress caused by growing up in an HD family and undergoing predictive genetic testing for HD (2), we included a group of community controls as well as a control group consisting of gene-negative subjects from HD families.

The first aim of this study was to investigate whether $C_{1} R$ availability is already decreased in pre-HD. Second, we wanted to investigate whether $\mathrm{CB}_{1} \mathrm{R}$ availability in pre-HD may correlate with specific psychiatric and cognitive symptoms.

\section{MATERIALS AND METHODS}

\section{Participants}

Fifteen pre-HD subjects (HTT CAG repeat length of 40 or more; absence of chorea or other motor signs typical of HD) were included in the study, as well as 15 gene-negative healthy controls from HD families (CAG repeat length of less than 36) and 12 healthy community 
controls (no personal or family history of neuropsychiatric disorders). We included the gene-negative control group in addition to the community control group to control for the distress of growing up in an HD family and undergoing predictive genetic testing for HD. Pre-HD subjects and gene-negative controls were recruited via the Department of Human Genetics of the University Hospitals Leuven after predictive genetic testing for HD. Community controls were recruited through advertisements in community newspapers and on a departmental website.

Subjects were excluded if they met any of the following criteria: past or current abuse of alcohol or other drugs, intake of psychotropic drugs, pregnancy, implanted devices or material that could interfere with the MRI scans, and previous radiation exposure of more than 1 $\mathrm{mSv}$ for other medical research studies during the past 12 mo.

All subjects underwent a general physical examination and blood and urine testing to exclude major internal pathologic states. Absence of any drug use at the time of $\mathrm{CB}_{1} \mathrm{R}$ PET scanning was confirmed by a urine stick test, which included toxicology for benzodiazepines, neuroleptics, opiates, cocaine and metabolites, amphetamines, and cannabinoids before the $\mathrm{CB}_{1} \mathrm{R}$ PET scan in all controls and pre-HD subjects.

The study was approved by the institutional Ethics Committee and was performed in accordance with the latest version of the World Medical Association Declaration of Helsinki. Written informed consent was obtained from all participants before the study.

\section{Clinical Assessments}

The motor part of the Unified Huntington's Disease Rating Scale was administered to all subjects on the day of PET. All subjects underwent a neuropsychologic test battery including the Mini-Mental State Examination, a test of episodic memory (Rey Auditory Verbal Learning Test), tests of attention and executive functioning (Digit Span Test and the Digit Symbol Subtest from the Wechsler Adult Intelligence Scale), Trail Making Tests A and B, the Stroop Test, the Letter Verbal Fluency and Animal Verbal Fluency Test, and tests of language (Boston Naming Test) and intelligence (Wechsler Adult Intelligence Scale).

Mood and behavioral symptoms were assessed using the Beck Depression Inventory, the State-Trait Anxiety Inventory, and the Problem Behavior Assessment for HD (PBA-HD) $(10,11)$. The PBA-HD is a semistructured interview specifically designed for assessment of behavioral problems in HD and comprises 3 subscales: PBA-HD apathy, PBA-HD irritability, and PBA-HD depression. Three of the 15 pre-HD subjects declined to undergo the PBA assessment.

The predicted age of motor onset in the pre-HD group was calculated according to Aylward et al. (12) as $\left[\left(-0.81 \times[C A G]_{n}\right)+\right.$ $(0.51 \times$ parental onset age $)+54.87]$. The 10 -y probability of disease onset was calculated using the model of Langbehn et al. (13). Disease burden was calculated as $[([\mathrm{CAG}] \mathrm{n}-35.5) \times$ age $](14)$.

\section{Image Acquisition and Brain Atrophy Assessment}

All subjects fasted for at least $4 \mathrm{~h}$ before PET imaging. They received 217.4 $\pm 68.7 \mathrm{MBq}$ (mean $\pm \mathrm{SD}$ ) of ${ }^{18} \mathrm{~F}-\mathrm{MK}-9470(8,15)$ as a slow intravenous injection under standard conditions (specific activity, $177.7 \pm 86.5 \mathrm{GBq} / \mu \mathrm{mol}$; injected mass dose, $3.7 \pm 0.3 \mu \mathrm{g}$ ). PET imaging was performed in 3-dimensional mode on a HiRez Biograph16 PET/CT camera (Siemens). At the beginning of each PET scan, a low-dose CT scan was conducted for attenuation correction purposes. An ${ }^{18}$ F-MK-9470 PET scan of 60 min was started $120 \mathrm{~min}$ after injection (16). Images were reconstructed using iterative 3dimensional ordered-subsets expectation maximization and were smoothed afterward with a 3-dimensional isotropic gaussian of $6 \mathrm{~mm}$ in full width at half maximum. All subjects also underwent high-resolution MRI on a 3-T scanner (Ingenia; Philips). Individual MR images were used to coregister PET data, to automatically and accurately delineate volumes of interest (VOIs), and to assess potential brain atrophy in patients both at the voxel level (by using voxel-based morphometry) and at the VOI level (by comparing groups' relative gray matter volumes [i.e., gray matter volume normalized to total intracranial volume] according to the 83-VOI N30R83 maximum-probability atlas of Hammers et al. (17)).

\section{Image Processing}

Parametric $\mathrm{CB}_{1} \mathrm{R}$ binding maps were calculated using the modified SUV [activity concentration $\times$ (subject's body weight $+70 / 2)$ /injected activity] (18), a validated noninvasive quantification method that gives a reliable estimate of the total distribution volume of ${ }^{18} \mathrm{~F}-\mathrm{MK}-9470$, as determined by full kinetic modeling in humans (16). The modified SUV measure was chosen for its decreased between-subject variability and therefore increased reproducibility and sensitivity to detect group differences compared with the fractional uptake ratio.

TABLE 1

Demographic and Clinical Characteristics of Study Subjects

\begin{tabular}{|c|c|c|c|}
\hline Characteristic & Community controls & Gene-negative controls & Pre-HD subjects \\
\hline Subjects & 12 & 15 & 15 \\
\hline \multicolumn{4}{|l|}{ Sex } \\
\hline Male & 6 & 9 & 8 \\
\hline Female & 6 & 6 & 7 \\
\hline Age $(y)$ & $39.9 \pm 15.1$ & $37.0 \pm 10.6$ & $39.3 \pm 9.9$ \\
\hline [CAG]n & NA & $20.33 \pm 2.92$ & $42.4 \pm 1.8$ \\
\hline UHDRS motor & $0.7 \pm 0.7$ & $1.3 \pm 1.1$ & $2.3 \pm 2.0$ \\
\hline Predicted age of onset (y) & - & - & $45.2 \pm 4.8$ \\
\hline $10-y$ probability of disease onset (\%) & - & - & $37.1 \pm 28.1$ \\
\hline Disease burden* & & & $264.3 \pm 74.6$ \\
\hline \multicolumn{4}{|c|}{$\begin{array}{l}{ }^{*} \text { Calculated as }([\mathrm{CAG}] \mathrm{n}-35.5) \times \text { age. } \\
\mathrm{NA}=\text { not available; UHDRS }=\text { Unified HD Rating Scale. } \\
\text { Qualitative data are expressed as } n \text {; continuous data are expressed as mean } \pm \text { SD. }\end{array}$} \\
\hline
\end{tabular}


TABLE 2

Neuropsychologic Assessments in Pre-HD Subjects and Controls

\begin{tabular}{|c|c|c|c|}
\hline Assessment & Control $^{\star}(n=27)$ & Pre-HD $(n=15)$ & $P$ \\
\hline \multicolumn{4}{|l|}{ Cognition } \\
\hline MMSE & $30.0(29.0-30.0)$ & $29.0(28.0-29.0)$ & 0.002 \\
\hline RAVLT total (A1-A5) & $55.8 \pm 8.6$ & $47.5 \pm 10.2$ & 0.008 \\
\hline RAVLT retention (A7) & $13.0(12.0-14.0)$ & $11.0(7.0-14.0)$ & 0.04 \\
\hline RAVLT recognition (A8) & $15.0(15.0-15.0)$ & $14.0(12.0-15.0)$ & 0.01 \\
\hline BNT & $56.0(53.0-58.0)$ & $56.0(53.0-58.0)$ & NS \\
\hline TMT A (s) & $25.0(23.0-34.0)$ & $31.0(18.0-38.0)$ & NS \\
\hline TMT B (s) & $52.5(47.5-63.0)$ & $74.0(54.0-99.0)$ & 0.004 \\
\hline Stroop word reading & $43.0(39.0-45.0)$ & $50.0(39.0-55.0)$ & NS \\
\hline Stroop color naming & $50.0(47.0-60.0)$ & $58.0(52.0-65.0)$ & 0.03 \\
\hline Stroop interference & $82.0(72.0-91.0)$ & $88.0(84.0-113.0)$ & 0.02 \\
\hline NART & $49.0(46.0-50.0)$ & $47.0(42.5-49.0)$ & NS \\
\hline DS forward & $6.0(6.0-7.0)$ & $6.0(5.0-7.0)$ & NS \\
\hline DS backward & $5.0(4.0-6.0)$ & $4.0(4.0-5.0)$ & 0.03 \\
\hline VF animal & $22.0(21.0-26.0)$ & $19.0(16.0-27.0)$ & NS \\
\hline VF N & $13.0(10.0-15.0)$ & $9.0(5.0-10.0)$ & 0.03 \\
\hline VF A & $13.0(11.0-15.0)$ & $8.0(6.0-10.0)$ & 0.007 \\
\hline VF K & $14.33 \pm 4.64$ & $11.53 \pm 6.31$ & NS \\
\hline Digit symbol & $85.0(76.0-93.0)$ & $64.0(54.0-88.0)$ & NS \\
\hline Block patrons & $67.0(65.0-68.0)$ & $66.0(57.0-67.0)$ & NS \\
\hline \multicolumn{4}{|l|}{ Mood and behavior } \\
\hline BDI & $1.0(0.0-5.0)$ & $4.0(1.0-10.0)$ & NS \\
\hline STAI 1 & $47.0(44.0-55.0)$ & $49.0(43.0-50.0)$ & NS \\
\hline STAI 2 & $47.8 \pm 3.9$ & $46.5 \pm 6.1$ & NS \\
\hline PBA-HD total & $2.7 \pm 3.1$ & $10.0 \pm 7.7$ & 0.002 \\
\hline PBA-HD apathy & $0.1 \pm 0.3$ & $1.4 \pm 2.0$ & 0.005 \\
\hline PBA-HD irritability & $0.4 \pm 0.6$ & $1.5 \pm 1.2$ & 0.01 \\
\hline PBA-HD depression & $0.9 \pm 1.3$ & $3.2 \pm 3.0$ & 0.02 \\
\hline
\end{tabular}

*Gene-negative and community controls.

MMSE = Mini-Mental State Examination; RAVLT = Rey Auditory Verbal Learning Test; BNT = Boston Naming Test; NS = not significant; TMT = Trail Making Test; NART = National Adult Reading Test; DS = Digit Span Test; VF N, A, K = verbal fluency for letters $\mathrm{N}, \mathrm{A}$, and $\mathrm{K} ; \mathrm{BDI}=$ Beck Depression Inventory; STAI = State-Trait Anxiety Inventory.

Skewed data are median followed by quartiles $1-3$ in parentheses. Normally distributed data are mean \pm SD. $P$ values are for results surviving Kruskal-Wallis test and Bonferroni test.

Further image processing was performed using an automatic pipeline in the PMOD brain PNEURO tool (PMOD, version 3.6; PMOD Technologies) (19). PET images were first rigidly coregistered to the corresponding volumetric T1-weighted MR images. The individual T1-weighted MR images were then nonlinearly coregistered to the standard Montreal Neurologic Institute space MRI template. Subsequently, the same was done for the PET images using the same spatial transformation as for the registered MR images. T1-weighted MR images were segmented into gray matter, white matter, and cerebrospinal fluid within native MRI space to generate a total of 83 individual VOIs according to the N30R83 atlas (17). Automatic delineation of the deep nuclei was performed by T1weighted MRI parcellation in the PMOD PNEURO tool. Partial-volume effect (PVE) correction was performed as implemented in PNEURO (20), using the methods of Müller-Gärtner et al. (21) and Rousset et al. (22) for the voxelwise and VOI-based analysis, respectively.

\section{Image Analysis and Statistics}

First, a voxelwise analysis was performed using Statistical Parametric Mapping (SPM8; Wellcome Department of Cognitive Neurology). PVE-corrected ${ }^{18} \mathrm{~F}-\mathrm{MK}-9470$ parametric maps were smoothed with a 10-mm isotropic gaussian kernel and were masked to exclude extracerebral activity. For analysis of absolute ${ }^{18} \mathrm{~F}-\mathrm{MK}-9470$ binding, no global normalization was used. $t$ maps were interrogated at a cluster-level $P$ value $\left(P_{\text {cluster }}\right)$ of less than 0.05 and a voxel-level $P$ value $\left(P_{\text {height }}\right)$ of less than 0.001 and with an extent threshold $\left(k_{\text {ext }}\right)$ of 50 voxels. A VOI-based group-comparison analysis was also performed using the N30R83 atlas. To avoid type II errors, VOIs were grouped into composite, bilateral VOIs for the frontal cortex, parietal cortex, lateral temporal cortex, medial temporal cortex, occipital cortex, anterior cingulate cortex, posterior cingulate cortex, and striatum. Because of its potential importance for the cognitive and behavioral 


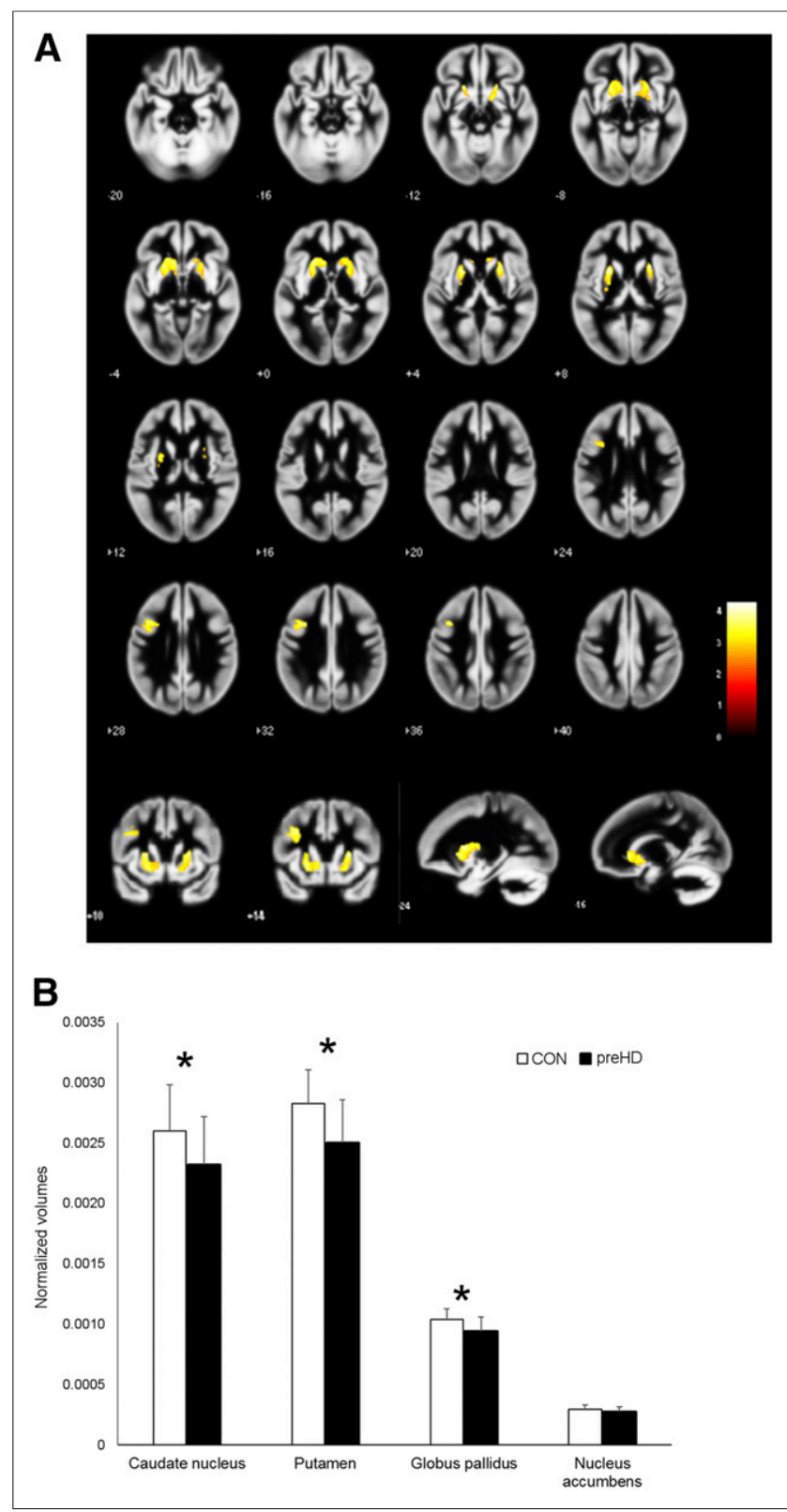

FIGURE 1. Decrease in striatal gray matter volume in pre-HD subjects in comparison with healthy controls $(\mathrm{CON})$ revealed at voxel level $\left(P_{\text {height }}<0.005\right)(\mathrm{A})$ and at VOI level $(\mathrm{B}) .{ }^{\star} P<0.05$.

measures in this study, the frontal cortex was also subdivided into prefrontal, dorsolateral prefrontal, ventrolateral prefrontal, ventromedial prefrontal, and orbitofrontal.

Statistical analyses were performed using Statistica (version 12; Statsoft Inc.) and SPSS (version 22.0; IBM). Shapiro-Wilk tests were used to test the distribution of variables against normality. ANOVA or the Kruskal-Wallis test, followed by a Bonferroni post hoc test, was used as appropriate.

To study the interrelatedness of the different behavioral and cognitive measures, we performed a factor analysis (principal components for the method extraction, 5 factors extracted, varimax normalized for the factor rotation).

Moreover, we investigated the degree to which regional $\mathrm{CB}_{1} \mathrm{R}$ availability in the pre-HD group was associated with cognitive and psychiatric measures when significantly different from controls, performing an
SPM correlation analysis $\left(P_{\text {cluster }}<0.05\right.$ and at a voxel-level $P_{\text {height }}<$ 0.001 , unless otherwise stated, with a $k_{\text {ext }}$ of 50 voxels) and a VOIbased analysis (Spearman rank test, $P<0.05$ ).

\section{RESULTS}

\section{Demographic and Clinical Characteristics}

In total, 15 pre-HD subjects, 15 gene-negative controls, and 12 community controls were included in the study (Table 1). Unified Huntington's Disease Rating Scale motor scores were slightly higher $(P=0.032)$ in pre-HD subjects than in community controls (Table 1), consistent with the concept that subtle motor changes can be detected on a group level in the pre-HD phase (5). There was no difference in age among the 3 groups (range, $P>0.64$ ). Moreover, no statistically significant difference in sex distribution was observed among the 3 groups ( $\chi^{2}$ test, $P=0.86$ ).

There were no differences between community controls and gene-negative controls in neuropsychologic scores, gray matter volume, or $\mathrm{CB}_{1} \mathrm{R}$ binding (Supplemental Table 1; Supplemental Figs. 1 and 2 [supplemental materials are available at http://jnm. snmjournals.org]). The community controls and gene-negative controls were therefore pooled into one control group for comparison with pre-HD subjects (Table 2).

Regarding the cognitive assessment, pre-HD subjects performed worse than controls on MMSE $(P=0.002)$, Rey Auditory Verbal

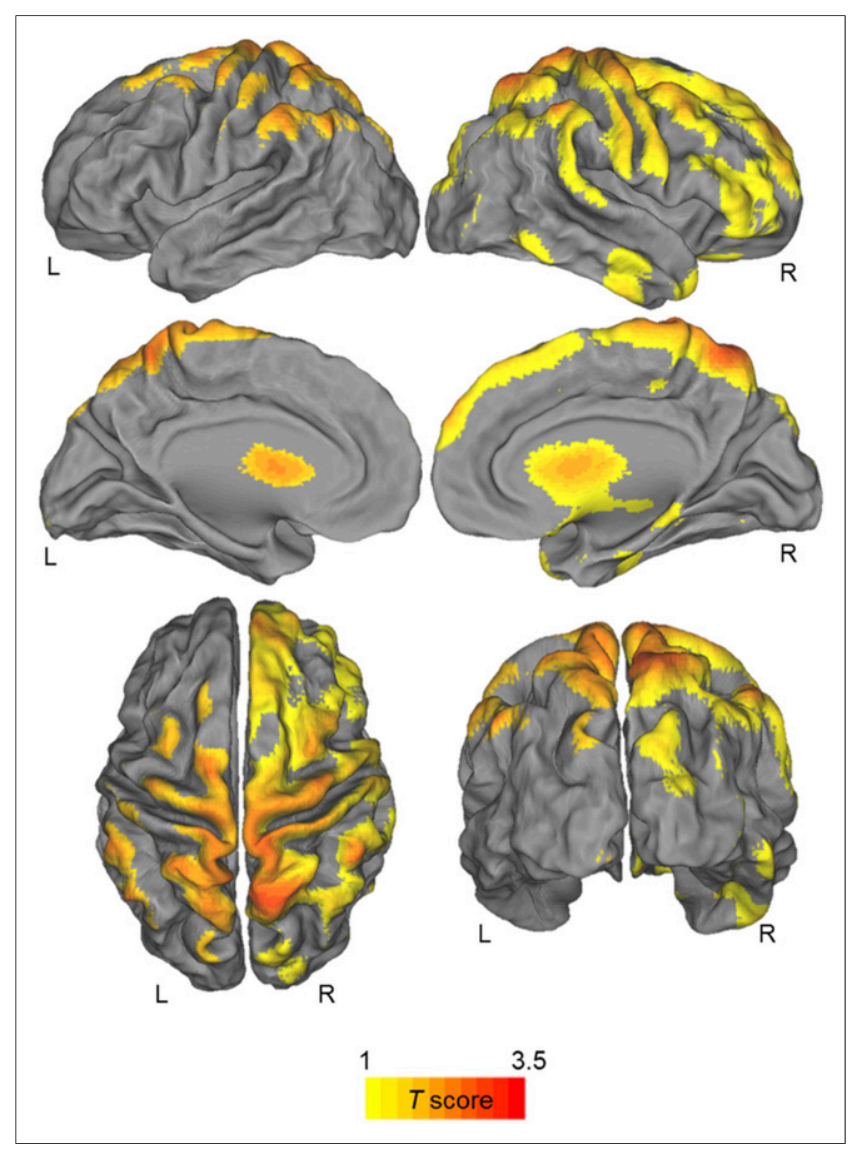

FIGURE 2. Statistical parametric $t$ map of significantly decreased $P V E$-corrected $\mathrm{CB}_{1} \mathrm{R}$ availability in pre-HD mutation carriers vs. controls. Maps are projected on PALS-B12 atlas (a population-averaged landmark- and surface-based atlas derived from 12 healthy young adults). Significance is shown with $t$ statistic color scale corresponding to level of significance at voxel level. 
Learning Test (range, $P=0.008-0.04$ ), verbal fluency (range, $P=$ 0.007-0.03), Stroop color naming $(P=0.03)$, and Stroop interference $(P=0.02)$ (Table 2$)$. As for mood and behavioral measures, pre-HD subjects had a significantly higher PBA-HD total score than controls $(P=0.002)$, as well as higher subscores (PBAHD apathy $[P=0.005]$, PBA-HD irritability $[P=0.01]$, and PBA-HD depression $[P=0.02]$ ) (Table 2 ). All reported $P$ values survived Bonferroni post hoc correction.

\section{Gray Matter Volume}

Voxel-based morphometry revealed significant striatal gray matter atrophy in pre-HD subjects bilaterally in the putamen, caudate nucleus, nucleus accumbens, and left middle frontal gyrus (Fig. 1A; Supplemental Table 2). These volumetric gray matter reductions in the pre-HD group were also observed at the VOI level by comparing relative VOIs (caudate: $-10.8 \%, P=0.03$; putamen: $-11.5 \%, P=0.002$; pallidum: $-9.5 \%, P=0.005$; nucleus accumbens: $-7.6 \%, P=0.065$; Fig. $1 \mathrm{~B})$. In pre-HD subjects, there were no significant associations between either the normalized volume decrease or the percentage decrease in gray matter volume and the cognitive and behavioral measures (nonparametric Spearman correlation test, $P<0.05$ ).

\section{$\mathrm{CB}_{1} \mathrm{R}$ Availability and Clinical Characteristics in Pre-HD}

VOI-based analysis showed no significant $\mathrm{CB}_{1} \mathrm{R}$ differences between the 2 control groups (gene-negative controls vs. community controls) in any region (average difference, $-1.0 \% \pm 1.8 \%$; range, $P=0.5-0.9$ ) (Supplemental Fig. 2). Therefore, also here the community control group was pooled with the gene-negative control group to create one control group for comparison of the ${ }^{18} \mathrm{~F}-\mathrm{MK}-9470$ data with the pre-HD group.

Compared with controls, the pre-HD group showed a decrease in PVE-corrected $\mathrm{CB}_{1} \mathrm{R}$ availability in the superior and middle prefrontal gyrus, superior parietal gyrus, precentral and posterior gyrus $\left(P_{\text {cluster }}=0.003\right.$ and $P_{\text {height }}=0.001$ for voxel level, $k_{\text {ext }}=$ 14449), and caudate nucleus $\left(P_{\text {cluster }}=0.016\right.$ and $P_{\text {height }}=0.001$ for voxel level, $k_{\mathrm{ext}}=205$ ) (Fig. 2). Similar results were obtained when the voxel-based analysis was performed with nonsmoothed PVE-corrected modified SUV images.

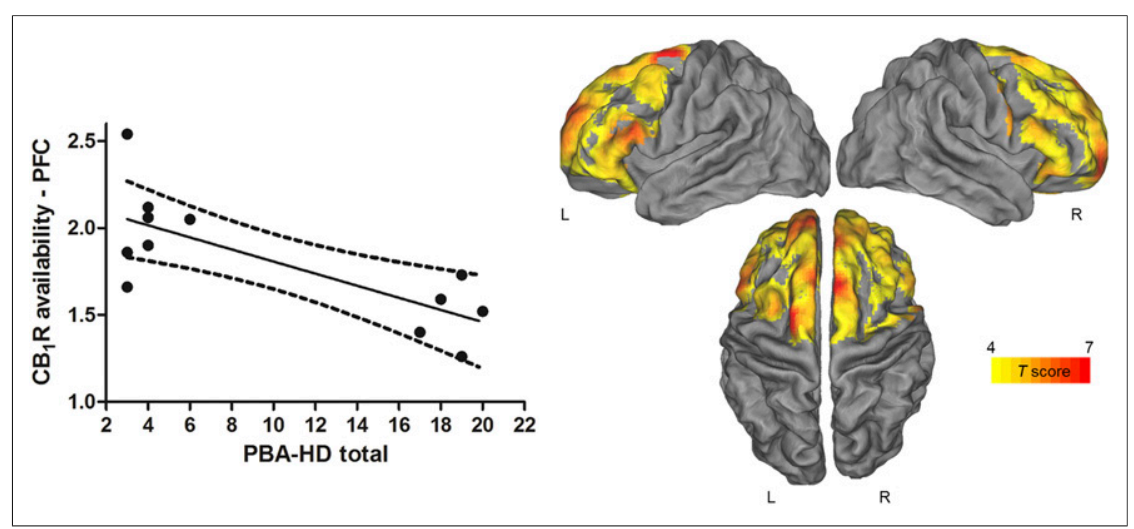

FIGURE 3. Negative correlation between $\mathrm{CB}_{1} \mathrm{R}$ and PBA-HD total in pre-HD. (Left) Scatterplot showing inverse correlation between individual PBA-HD total scores and $\mathrm{CB}_{1} R$ availability in prefrontal cortex (PFC) at VOI-based level. Solid line represents regression line, and dotted lines represent 95\% confidence interval. (Right) Statistical parametric maps showing inverse correlation between PVE-corrected $\mathrm{CB}_{1} \mathrm{R}$ availability in pre-HD and PBA-HD total. Maps are projected on PALS-B12 atlas (a population-averaged landmark- and surface-based atlas derived from 12 healthy young adults). Significance is shown with $t$ statistic color scale corresponding to level of significance at voxel level.
Correlations with PVE-corrected $\mathrm{CB}_{1} \mathrm{R}$ availability were found for mood and behavioral assessments but not for cognitive measures. We found a negative correlation $\left(P_{\text {cluster }}<0.001\right)$ between the total PBA-HD score and the PVE-corrected $\mathrm{CB}_{1} \mathrm{R}$ availability in a bilateral cluster located in the frontal lobe, including the precentral gyrus $\left([x, y, z]=[64,10,30], t=9.17, P_{\text {height }}=\right.$ $3.7 \cdot 10^{-6}$ at voxel level); inferior, middle, and superior frontal gyrus, orbitofrontal gyrus; and anterior cingulate cortex (Fig. 3, right). Additionally, the SPM analysis showed that PBA-HD depression factor was inversely correlated with the PVE-corrected $\mathrm{CB}_{1} \mathrm{R}$ availability in a cluster at the $P_{\text {height }}$ level of 0.005 , located mainly across the superior frontal gyrus $([x, y, z]=[-32,-2,64]$, $t=5.94, P_{\text {height }}=1.1 \cdot 10^{-4}$ at voxel level) (Fig. 4, right).

These findings were largely confirmed at a VOI level. Total PBA-HD scores showed an inverse correlation with $\mathrm{CB}_{1} \mathrm{R}$ availability in all prefrontal regions (range, $r=-0.64$ to -0.72 ; range, $P=0.02-0.008$; Fig. 3, left) and cingulate cortex (posterior cingulate cortex: $r=-0.60, P=0.04$; anterior cingulate cortex: $r=$ $-0.69, P=0.01)$ of pre-HD subjects. Regarding the PBA-HD subscores, only the correlation between the PBA-HD depression score and $\mathrm{CB}_{1} \mathrm{R}$ availability reached significance in the prefrontal cortex $(r=-0.61, P=0.03$; Fig. 4, left), orbitofrontal cortex, and anterior cingulate cortex $(r=-0.59, P=0.04)$ (PBA-HD apathy: range, $P=0.09-0.21$; PBA-HD irritability: range, $P=$ $0.26-0.59)$.

Significant factor loadings were found for the Beck Depression Inventory score, State-Trait Anxiety Inventory 2, Total PBA-HD, and PBA-HD apathy and depression (range of marked loadings, 0.74-0.88), showing that mood and motivation symptoms correlated with each other in the pre-HD group. Individual scores for factor 1 were intercorrelated with the $\mathrm{CB}_{1} \mathrm{R}$ availability in all prefrontal cortex regions (range, $r=0.60-0.69 ; P=0.013-$ $0.41)$ and anterior cingulate cortex $(r=0.67, P=0.018)$ (Supplemental Fig. 3).

$\mathrm{CB}_{1} \mathrm{R}$ availability did not correlate with [CAG]n (SPM correlation analysis with $[\mathrm{CAG}] \mathrm{n}$ as covariate and age as nuisance variable), even at a liberal $P_{\text {height }}$ threshold of less than 0.01 (uncorrected). There were no significant correlations between disease burden and either regional $\mathrm{CB}_{1} \mathrm{R}$ levels (range, $P=0.27-0.97$ ) or PBA-HD scores (range, $P=0.25-0.75$ ) in the pre-HD group.

\section{DISCUSSION}

Psychiatric symptoms are common in pre-HD (1-0), but the underlying brain changes are poorly understood. To our knowledge, our PET study is the first to find a molecular correlate of behavioral symptoms in pre-HD.

In contrast to the more global decrease in cerebral $\mathrm{CB}_{1} \mathrm{R}$ availability seen in manifest $\mathrm{HD}$ patients (8), we found regionally restricted $C_{1} R$ reductions in our pre-HD group in the superior and middle prefrontal gyrus, precentral gyrus, and caudate nucleus. These regional $\mathrm{CB}_{1} \mathrm{R}$ reductions in pre-HD are unlikely to be caused by the psychologic stress associated with growing up in an HD family and undergoing predictive genetic testing, because there was 


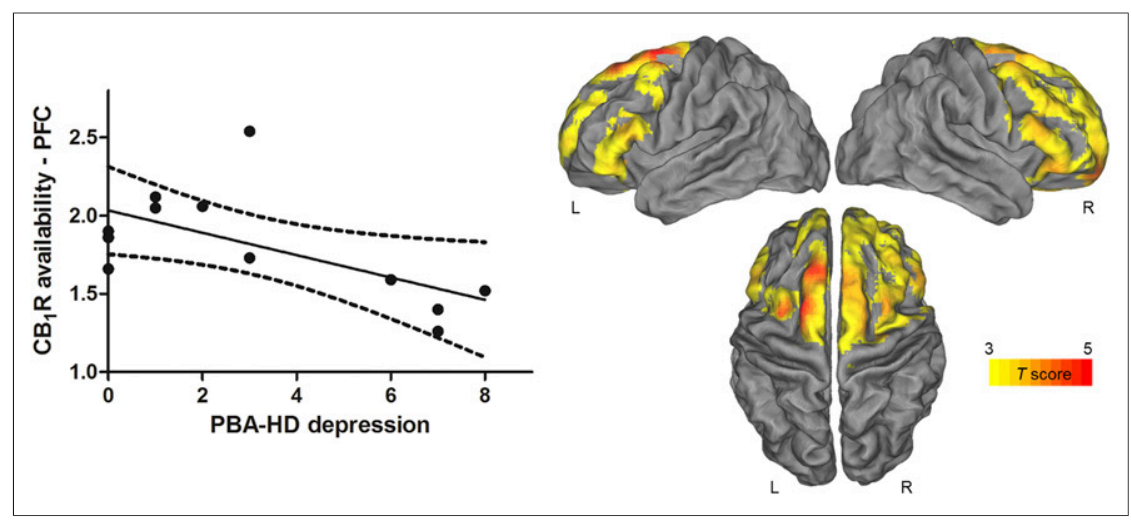

FIGURE 4. Negative correlation between $\mathrm{CB}_{1} \mathrm{R}$ and PBA-HD depression in pre-HD. (Left) Scatterplot shows inverse correlation between individual PBA-HD depression scores and $\mathrm{CB}_{1} \mathrm{R}$ availability in prefrontal cortex (PFC) at VOI-based level. Solid line represents regression line, and dotted lines represent $95 \%$ confidence interval. (Right) Statistical parametric maps and inverse correlation between PVE-corrected $\mathrm{CB}_{1} \mathrm{R}$ availability in pre-HD mutation carriers with PBA-HD depression. Maps are projected on PALS-B12 atlas (a population-averaged landmark- and surface-based atlas derived from 12 healthy young adults). Significance is shown with $t$ statistic color scale corresponding to level of significance at voxel level.

no difference in $\mathrm{CB}_{1} \mathrm{R}$ availability between gene-negative controls and community controls. No differences were found in $\mathrm{CB}_{1} \mathrm{R}$ availability levels, cognitive measures, or psychiatric symptoms between the 2 control groups, indicating that growing up with affected and at-risk family members and undergoing predictive genetic testing for $\mathrm{HD}$ did not have any detectable influence on $\mathrm{CB}_{1} \mathrm{R}$ signaling and cognitive and behavioral measures. Instead, the $C_{1} R$ reductions in pre-HD may be due to a direct repressive effect of mutant huntingtin on $\mathrm{CB}_{1} \mathrm{R}$ gene transcription $(23,24)$.

Importantly, we found $\mathrm{CB}_{1} \mathrm{R}$ in the prefrontal and cingulate cortex to have an inverse correlation with behavioral symptoms, in particular depression. The prefrontal cortex and anterior cingulate cortex are involved in emotion regulation via reciprocal connections with limbic structures and play a major role in the pathophysiology of mood disorders $(25,26)$. Moreover, a large body of preclinical and human data suggest an important role for hypofunction of the endocannabinoid system, particularly in the prefrontal cortex, in the pathogenesis of depression $(27,28)$. Genetic deletion or pharmacologic inhibition of $\mathrm{CB}_{1} \mathrm{R}$ in rodents induces symptoms that mimic human depression $(27,29)$. Conversely, activation of endocannabinoid transmission has antidepressantlike properties in established animal models of depression $(30,31)$. Finally, clinical trials showed that the $\mathrm{CB}_{1} \mathrm{R}$ inverse agonist rimonabant increases the risk of depressed mood $(32,33)$.

Although we did not find a significant correlation between PBA-HD apathy and $\mathrm{CB}_{1} \mathrm{R}$ availability, there was a strong intercorrelation between test scores for depression and apathy using factor analysis, suggesting that apathy and depression are difficult to tease apart in pre-HD and that the neurodegenerative process disrupts circuits involved in both mood and motivation at an early stage.

If the frontal $\mathrm{CB}_{1} \mathrm{R}$ reduction causally contributes to the behavioral symptoms in HD mutation carriers, pharmacologic endocannabinoid system activation could have beneficial effects on these symptoms. Interestingly, a pilot study with the $\mathrm{CB}_{1} \mathrm{R}$ agonist nabilone in HD patients found preliminary evidence for improvement of neuropsychiatric symptoms (including depression and apathy) (34).
Some limitations of the present study should be acknowledged. First, the pre-HD group was relatively small and needs independent replications in larger pre-HD populations. Second, longitudinal followup studies in the pre-HD cohort will be required to assess the potential value of $\mathrm{CB}_{1} \mathrm{R}$ PET as a biomarker for pre-HD progression in comparison with other imaging modalities such as volumetric MRI (35) and PDE10A PET (36-38). Finally, larger clinical studies are warranted to investigate the effects of activators of endocannabinoid signaling on behavioral symptoms in pre-HD and HD.

\section{CONCLUSION}

The principal findings of this PET study are the decreased $\mathrm{CB}_{1} \mathrm{R}$ availability in the prefrontal cortex in pre-HD mutation carriers and the strong inverse correlation between frontal $\mathrm{CB}_{1} \mathrm{R}$ levels in pre-HD and behavioral symptoms, in particular scores on depression. Taken together, the association between behavioral symptoms and reduced prefrontal $\mathrm{CB}_{1} \mathrm{R}$ levels may provide new insight into the molecular basis of neuropsychiatric symptoms in pre-HD and suggest new therapeutic avenues targeting the endocannabinoid system.

\section{DISCLOSURE}

Jenny Ceccarini is a postdoctoral fellow of the Research FundFlanders. Wim Vandenberghe and Koen Van Laere are Senior Clinical Investigators of the Research Fund-Flanders. No other potential conflict of interest relevant to this article was reported.

\section{ACKNOWLEDGMENTS}

We thank Prof. Dr. Jean-Pierre Frijns, Prof. Dr. Annick Vogels, and Andrea Bogaerts for assistance in recruiting participants, and we thank the PET radiopharmacy department and Kwinten Porters for their skilled help in tracer production and data acquisition.

\section{REFERENCES}

1. Duff K, Paulsen JS, Beglinger LJ, Langbehn DR, Stout JC; Predict-HD Investigators of the Huntington Study Group. Psychiatric symptoms in Huntington's disease before diagnosis: the predict-HD study. Biol Psychiatry. 2007;62:1341-1346.

2. Julien CL, Thompson JC, Wild S, et al. Psychiatric disorders in preclinical Huntington's disease. J Neurol Neurosurg Psychiatry. 2007;78:939-943.

3. Marshall J, White K, Weaver M, et al. Specific psychiatric manifestations among preclinical Huntington disease mutation carriers. Arch Neurol. 2007;64:116-121.

4. Martinez-Horta S, Perez-Perez J, van Duijn E, et al. Neuropsychiatric symptoms are very common in premanifest and early stage Huntington's disease. Parkinsonism Relat Disord. 2016;25:58-64.

5. Ross CA, Aylward EH, Wild EJ, et al. Huntington disease: natural history, biomarkers and prospects for therapeutics. Nat Rev Neurol. 2014;10:204-216.

6. Van den Stock J, De Winter FL, Ahmad R, et al. Functional brain changes underlying irritability in premanifest Huntington's disease. Hum Brain Mapp. 2015;36:2681-2690.

7. Lu HC, Mackie K. An introduction to the endogenous cannabinoid system. Biol Psychiatry. 2016;79:516-525.

8. Van Laere K, Casteels C, Dhollander I, et al. Widespread decrease of type 1 cannabinoid receptor availability in Huntington disease in vivo. $\mathrm{J} \mathrm{Nucl} \mathrm{Med}$. 2010;51:1413-1417. 
9. Ooms M, Rietjens R, Rangarajan JR, et al. Early decrease of type 1 cannabinoid receptor binding and phosphodiesterase 10A activity in vivo in R6/2 Huntington mice. Neurobiol Aging. 2014;35:2858-2869.

10. Kingma EM,van Duijn E, Timman R, van der Mast RC, Roos RA. Behavioural problems in Huntington's disease using the problem behaviours assessment. Gen Hosp Psychiatry. 2008;30:155-161.

11. Craufurd D, Thompson JC, Snowden JS. Behavioral changes in Huntington disease. Neuropsychiatry Neuropsychol Behav Neurol. 2001;14:219-226.

12. Aylward EH, Codori AM, Barta PE, Pearlson GD, Harris GJ, Brandt J. Basal ganglia volume and proximity to onset in presymptomatic Huntington disease. Arch Neurol. 1996;53:1293-1296.

13. Langbehn DR, Brinkman RR, Falush D, Paulsen JS, Hayden MR; International Huntington's Disease Collaborative Group. A new model for prediction of the age of onset and penetrance for Huntington's disease based on CAG length. Clin Genet. 2004;65:267-277.

14. Penney JB Jr, Vonsattel JP, MacDonald ME, Gusella JF, Myers RH. CAG repeat number governs the development rate of pathology in Huntington's disease. Ann Neurol. 1997;41:689-692.

15. Burns HD, Van Laere K, Sanabria-Bohorquez S, et al. ${ }^{18} \mathrm{~F}-\mathrm{MK}-9470$, a positron emission tomography (PET) tracer for in vivo human PET brain imaging of the cannabinoid-1 receptor. Proc Natl Acad Sci USA. 2007;104:9800-9805.

16. Sanabria-Bohórquez SM, Hamill TG, Goffin K, et al. Kinetic analysis of the cannabinoid-1 receptor PET tracer $\left[{ }^{18} \mathrm{~F}\right] \mathrm{MK}-9470$ in human brain. Eur J Nucl Med Mol Imaging. 2010;37:920-933.

17. Hammers A, Koepp MJ, Richardson MP, Hurlemann R, Brooks DJ, Duncan JS. Grey and white matter flumazenil binding in neocortical epilepsy with normal MRI: a PET study of 44 patients. Brain. 2003;126:1300-1318.

18. Thie JA, Hubner KF, Isidoro FP, Smith GT. A weight index for the standardized uptake value in 2-deoxy-2-[F-18]fluoro-D-glucose-positron emission tomography. Mol Imaging Biol. 2007;9:91-98.

19. Brendel M, Pogarell O, Xiong G, et al. Depressive symptoms accelerate cognitive decline in amyloid-positive MCI patients. Eur J Nucl Med Mol Imaging. 2015;42:716-724.

20. Brendel M, Hogenauer M, Delker A, et al. Improved longitudinal $\left[{ }^{18} \mathrm{~F}\right]$-AV45 amyloid PET by white matter reference and VOI-based partial volume effect correction. Neuroimage. 2015;108:450-459.

21. Müller-Gärtner HW, Links JM, Prince JL, et al. Measurement of radiotracer concentration in brain gray matter using positron emission tomography: MRI-based correction for partial volume effects. J Cereb Blood Flow Metab. 1992;12:571-583.

22. Rousset OG, Ma Y, Evans AC. Correction for partial volume effects in PET: principle and validation. J Nucl Med. 1998;39:904-911.

23. Kuhn A, Goldstein DR, Hodges A, et al. Mutant huntingtin's effects on striatal gene expression in mice recapitulate changes observed in human Huntington's disease brain and do not differ with mutant huntingtin length or wild-type huntingtin dosage. Hum Mol Genet. 2007;16:1845-1861.

24. McCaw EA, Hu H, Gomez GT, Hebb AL, Kelly ME, Denovan-Wright EM. Structure, expression and regulation of the cannabinoid receptor gene (CB1) in Huntington's disease transgenic mice. Eur J Biochem. 2004;271:4909-4920.

25. Maletic V, Robinson M, Oakes T, Iyengar S, Ball SG, Russell J. Neurobiology of depression: an integrated view of key findings. Int J Clin Pract. 2007;61:20302040.

26. Whittle S, Allen NB, Lubman DI, Yucel M. The neurobiological basis of temperament: towards a better understanding of psychopathology. Neurosci Biobehav Rev. 2006;30:511-525.

27. Hillard CJ, Liu QS. Endocannabinoid signaling in the etiology and treatment of major depressive illness. Curr Pharm Des. 2014;20:3795-3811.

28. McLaughlin RJ, Hill MN, Gorzalka BB. A critical role for prefrontocortical endocannabinoid signaling in the regulation of stress and emotional behavior. Neurosci Biobehav Rev. 2014;42:116-131.

29. Valverde O, Torrens M. CB1 receptor-deficient mice as a model for depression. Neuroscience. 2012;204:193-206.

30. Adamczyk P, Golda A, McCreary AC, Filip M, Przegalinski E. Activation of endocannabinoid transmission induces antidepressant-like effects in rats. $J$ Physiol Pharmacol. 2008;59:217-228.

31. Gobbi G, Bambico FR, Mangieri R, et al. Antidepressant-like activity and modulation of brain monoaminergic transmission by blockade of anandamide hydrolysis. Proc Natl Acad Sci USA. 2005;102:18620-18625.

32. Christensen R, Kristensen PK, Bartels EM, Bliddal H, Astrup A. Efficacy and safety of the weight-loss drug rimonabant: a meta-analysis of randomised trials. Lancet. 2007;370:1706-1713.

33. Topol EJ, Bousser MG, Fox KA, et al. Rimonabant for prevention of cardiovascular events (CRESCENDO): a randomised, multicentre, placebo-controlled trial. Lancet. 2010;376:517-523.

34. Curtis A, Mitchell I, Patel S, Ives N, Rickards H. A pilot study using nabilone for symptomatic treatment in Huntington's disease. Mov Disord. 2009;24:22542259.

35. Aylward EH. Magnetic resonance imaging striatal volumes: a biomarker for clinical trials in Huntington's disease. Mov Disord. 2014;29:1429-1433.

36. Ahmad R, Bourgeois S, Postnov A, et al. PET imaging shows loss of striatal PDE10A in patients with Huntington disease. Neurology. 2014;82:279-281.

37. Russell DS, Barret O, Jennings DL, et al. The phosphodiesterase 10 positron emission tomography tracer, ${ }^{18} \mathrm{~F}-\mathrm{MNI}-659$, as a novel biomarker for early Huntington disease. JAMA Neurol. 2014;71:1520-1528.

38. Niccolini F, Haider S, Reis Marques T, et al. Altered PDE10A expression detectable early before symptomatic onset in Huntington's disease. Brain. 2015;138: 3016-3029. 